Bishop, Pace and Spiegelman have shown that the 5 terminal base of the minus strand of $Q \beta$ made in vitro is-like that of the plus strand- $\mathrm{G}$ and the $3^{\prime}$ terminal base of both strands is $\mathrm{C}$. Why then is the 3 ' terminal base of plus strand RNA isolated from intact phage $A$, not $C$ ? It seems that an enzyme must add at least the terminal $A$ and possibly the CCA sequence to the $3^{\prime}$ end of $Q \beta$ RNA. The search is now on for the enzyme. Whether it is similar, identical or totally unrelated to the enzyme which adds CCA to $t$ RNA remains to be seen. And what is the function of the $3^{\prime}$ terminal A ? Does it protect the RNA from exonucleases?

At the other end of the molecule, the $5^{\prime}$ terminal base of all the phage RNAs so far analysed is triphosphoryl. ated $\mathrm{G}$ (pppGpXpYp), whereas other cellular RNAs, for example, $5 S$ RNA, the sequence of which has just been published in full by Brownlee, Sanger and Barell (J. Mol. Biol., 34, 379; 1968), have 5' monophosphate groups. Because RNA is polymerized in the $5^{\prime} \rightarrow 3^{\prime}$ direction from nucleoside triphosphates, all RNA molecules are presumably made with a triphosphorylated $5^{\prime}$ terminus which must subsequently be cleaved by a dephosphorylase. Wimmer and Reichmann (Science, 160, 1452 ; 1968) have produced new evidence to support this idea. They have found that the $5^{\prime}$ terminal sequence of RNA from Satcllite Tobacco Necrosis Virus is ppApGpUp. The terminal A has a $5^{\prime}$ pyrophosphate group in contrast to the triphosphate group of RNAphage RNA and the monophosphate of cellular RNAs. There must therefore be an enzyme or enzymes in tobacco plants capable of removing the $\gamma$ phosphate group of a $5^{\prime}$ triphosphate just as there is in $E$. coli.

\section{Transfer Factors}

\section{from our Molecular Biology Correspondent}

EvIDExCE has accrued from several laboratories that the transfer of amino-acids from amino-acyl- $t$ RNA to a growing peptide chain requires the presence of a nucleotide (GTP) and certain proteins from the supernatant. Many data on the nature and activity of these substances have been brought together in the current issue of Arch. Biochem., which is dedicated to the late Richard Schweet and includes several papers from his own laboratory.

So far, three factors appear to be generally agreed on, but several different terminologies are in use. Thus Ravel et al. (Arch. Biochem., 125, 514; 1968) describe the preparation of three fractions, $F-I A$, $F-I B$ and $F-I I$ from $E$. coli by ion exchange chromatography. It is now recognized that amino-acyl- $t$ RNA has two modes of interaction with the ribosomemessenger complex, one enzymic, the other nonenzymic, and the former process requires GTP and either $F-I A$ or $F-I B$. Unlike the non-enzymic binding process, this reaction is not inhibited by $t \mathrm{RNA}$ stripped of its amino-acid. Skogerson and Moldave (ibid., 497) have made further observations, using puromycin, which becomes one partner in a peptide bond: GTP and their "transferase- $I$ " are involved in attachment of the amino-acyl-tRNA, but the peptide bond to puromycin is then formed without the intervention of either. It is suggested that the ribosome itself bcars a peptide bond-synthetase. The second supernatant factor, transferase-II, together with GTT, is found in normal peptide synthesis to catalyse the transfer of the new peptidyl-tRNA to the other ribosomal binding site, in preparation for the next cycle ("translocase"). Transfer factors from $E$. coli, termed $T$ and $G$, were also prepared by Lipmann and his associates, and $T$ has been further separated into two fractions, $T_{u}$ and $T_{s}$ (unstable and stable, respectively), which clearly correspond to the $F-I A$ and $F-I B$ of Ravel et al. Felicetti and Lipmann (ibid., 548) now note a similar pattern in mammalian systems (rat liver and rabbit reticulocyte), with two fractions $T_{1}$ and $T_{2}$, corresponding to $T$ and $G$, the second associated with GTPase activity.

The role of supernatant fractions in initiation of protein synthesis has been studied in Ochoa's laboratory (Iwasaki et al., ibid., 542) in the E. coli system. Three fractions have again been prepared, $F_{1}, F_{2}$ and $F_{3}$. $F_{3}$ is said to be required for the attachment of natural messenger ( $Q \beta$-phage $\mathrm{RNA}$ ) to the ribosome; $F_{2}$ then brings about the binding of formylmethionyl$t$ RNA, so that synthesis ean begin. $F_{1}$ is also required at this stage. A synthetic messenger, bearing the initiation codon at the $5^{\prime}$-end, will bind to the ribosome, however, without the aid of $F_{3}$, and $F_{1}$ can then similarly be dispensed with. Another supernatant protein, required for initiation of haemoglobin synthesis, though not propagation, has been isolated by Miller and Schweet (ibid., 632).

The atypical nature of synthetic messengers also emerges from the work of Arlinghaus et al. (ibid., 604); in the reticulocyte system some 70 per cent of labelled amino-acids appear in acid-precipitable polypeptide chains, and the remainder in the acid-soluble fraction, consisting of small peptides. With poly- $U$ as messenger, on the other hand, there is little incorporation into acid-insoluble material, and it is inferred that chain initiation is favoured relative to the polymerization reaction.

In another paper from Schweet's laboratory (Schaeffer et al., ibid., 614) the binding of phenylalanyl-tRNA to the poly-U ribosome complex has been studied. In the best conditions for polypeptide synthesis, GTP and the usual transfer fraction were required for binding, but under aberrant conditions (high magnesium and low potassium concentrations) these ceased to be necessary. On incubation with the peptide synthetase fraction, a dipeptide (phe.phe) is produced in the former case, but for the non-enzymically bound $t$ RNA to yield a dipeptide, GTP and the binding factor are expressly needed. An explanation is suggested in terms of binding at both $t$ RNA sites under the anomalous conditions. These results may be contrasted with those of Pestka (J. Biol. Chem., 243,$2810 ; 1968$ ) who has described the formation of short peptides, primarily phe.phe, in the absence of supernatant enzymes or GTP, in the $E$. coli system. This process has a characteristic response to magnesium concentration. The points of difference from the results of Schaeffer et al. may represent a real difference between bacterial and mammalian ribosomes, and indeed it has been reported that, in reticulocytes, initiation does not require a formylated amino-acyl$t$ RNA. Pestka's observations recall also the notion that the peptide synthetase is an integral part of the ribosome.

It may be noted that two transfer factors have recently been purified and crystallized (Parmeggiani, Biochem. Biophys. Res. Commun., 30, 613; 1968). These are the $G$ and $T$ factors of Lipmann. They sediment at $5 \cdot 3$ and $5 \cdot 18$, respectively. 